\title{
Operators in ultraviolet completions for Electroweak collective symmetry breaking
}

\author{
Nobuhiro Uekusa \\ Department of Physics, Osaka University, Toyonaka, Osaka 560-0043 Japan
}

\begin{abstract}
In a gauge theory with $\mathrm{SU}(5)$ broken to $\mathrm{SU}(2) \times \mathrm{U}(1)$, the Higgs mass squared receives only logarithmic divergence from all scalar-gauge interactions at one loop. The same pattern of gauge symmetry breaking can be achieved without any scalar fields in a five-dimensional model.
\end{abstract}

Keywords: Higgs mass, Collective symmetry breaking, Extra dimensions PACS: $12.60 . \mathrm{Cn}$

OU-HET 641/2009

Quadratic divergence is one of the fundamental problems of the standard model of particle physics. The structure of quantum loop corrections is different between scalars and fermions. It is chiral symmetry that protects fermions from having quadratic divergence. Scalar fields do not have such a symmetry in the standard model. Applying symmetry principle to scalar fields has been a clue to understand physics beyond the standard model. If scalar fields were Nambu-Goldstone bosons in broken global symmetry, they are exactly massless and it is led to little Higgs scenarios [1,2].

In little Higgs scenarios, if gauge and Yukawa couplings are vanishing, Higgs fields have derivative couplings and they have shift symmetry so that there is no potential. The scalar fields are described in a non-linear sigma model. The assumption that the global symmetry is explicitly broken only when two or more couplings are non-vanishing leads to a potential with at most logarithmic divergence. This collective symmetry breaking mechanism has been studied in many papers $[3,4,5,6]$. In the littlest Higgs model, the resulting operator of scalar-gauge interactions is, for example,

$$
W_{1 \mu} W_{2}^{\mu} h^{\dagger} h
$$

where $h$ is the Higgs field and $W_{1 \mu}$ and $W_{2 \mu}$ are $[\mathrm{SU}(2)]^{2}$ gauge bosons. At one loop, the gauge bosons do not produce the quadratic divergence for the Higgs mass squared through the interaction (1). While the collective symmetry breaking mechanism requires two or more couplings, such a group as $[\mathrm{SU}(2)]^{2}$ could be a subgroup of a single group. It should be clarified whether operators such as Eq. (1) can be derived in a renormalizable gauge theory with a single large group broken to two or more subgroups.

Recently, in Ref. [7] a weakly-coupled renormalizable ultraviolet completion of a little Higgs scenario was proposed. It was claimed that heavy modes are integrated out and that the remaining theory is a non-linear sigma model due to the Coleman-Wess-Zumino theorem $[8,9]$. In the model, the Higgs mass squared receives radiative correction of the order of $100 \mathrm{GeV}$ and the non-linear sigma model has the decay constant $f \sim 1 \mathrm{TeV}$ and the ultraviolet momentum cutoff $\Lambda \sim 10 \mathrm{TeV}$. Also, in an explicit example, several effective couplings with the form (1) were shown. To pursue this possibility of high 
energy theory for no quadratic divergence as an extension of the collective symmetry breaking mechanism, it would be important to examine more couplings.

In the following, we give all the couplings of scalar-gauge interactions in a weaklycoupled gauge theory beyond the ultraviolet momentum cutoff of models where the collective symmetry breaking mechanism protects the Higgs mass squared against having quadratic divergence. As another aspect, we study a possibility of gauge symmetry breaking by boundary conditions without any scalar fields.

We start with a renormalizable gauge theory with a single group which is broken by the vacuum expectation values of scalar fields, aiming for having scalar-gauge interactions of the form

$$
g^{2} W_{1 \mu} W_{2}^{\mu} h^{\dagger} h
$$

where a gauge coupling is denoted as $g$. For the interaction (2), the one-loop diagram for the Higgs mass squared has propagators of two gauge bosons. Therefore the divergence is at most logarithmic. Since Eq. (2) is written as

$$
g^{2}\left(\frac{W_{1 \mu}+W_{2 \mu}}{\sqrt{2}}\right)^{2} h^{\dagger} h-g^{2}\left(\frac{W_{1 \mu}-W_{2 \mu}}{\sqrt{2}}\right)^{2} h^{\dagger} h,
$$

the absence of the quadratic divergence for the Higgs mass squared is also regarded as a cancellation. In little Higgs models, the collective symmetry breaking mechanism can produce the operator (2). In such a model, the self-interaction of the Higgs fields obeys a non-linear sigma model. The Lagrangian terms of expansion at lower order are $\left|\partial_{\mu} h\right|^{2}+f^{-2}\left|\partial_{\mu} h\right|^{2} h^{\dagger} h$. The quadratic divergence of one-loop contribution to the kinetic term is $\Lambda^{2} /\left(16 \pi^{2} f^{2}\right)$, where $\Lambda$ is the ultraviolet momentum cutoff. For the nonlinear sigma model to be viable, the cutoff has the upper bound $\Lambda<4 \pi f$. The dominant correction to the Higgs mass squared is $\left(g^{4} /\left(16 \pi^{2}\right)\right) f^{2} \log \left(\Lambda^{2} / f^{2}\right)$. If the correction is of the order of $100 \mathrm{GeV}$, the decay constant is obtained as $f \sim 1 \mathrm{TeV}$ and then $\Lambda \sim 10 \mathrm{TeV}$. Thus our starting theory is defined at higher scales than $10 \mathrm{TeV}$.

We choose the single gauge group as SU(5). The gauge symmetry is broken by the vacuum expectation values of two types of scalar fields. We assume that these two sectors yield separate gauge symmetry breakings

$$
\mathrm{SU}(5) \rightarrow[\mathrm{SU}(2) \times \mathrm{U}(1)]^{2}, \quad \mathrm{SU}(5) \rightarrow \mathrm{SO}(5) .
$$

The Higgs fields are included in the sector of the breaking $\mathrm{SU}(5) \rightarrow \mathrm{SO}(5)$. The scalar fields in the other sector are decoupled to the Higgs fields. The scalar fields we discuss are only in the sector of $\mathrm{SU}(5) \rightarrow \mathrm{SO}(5)$.

The scalar field responsible for the breaking $\mathrm{SU}(5) \rightarrow \mathrm{SO}(5)$ is a scalar $\underline{15}$ which transforms as a complex symmetric matrix $S \rightarrow S^{\prime}=U S U^{T}$ under SU(5). The potential is written as $V=-M^{2} \operatorname{Tr}\left[S S^{\dagger}\right]+\lambda_{1}\left(\operatorname{Tr}\left[S S^{\dagger}\right]\right)^{2}+\lambda_{2} \operatorname{Tr}\left[\left(S S^{\dagger}\right)^{2}\right]$, where $M, \lambda_{1}$ and $\lambda_{2}$ are the coupling constants. From the stationary condition, the nonzero expectation value is given by $S S^{\dagger}=f_{S}^{2} \mathbf{1}_{5}$ (for $10 \lambda_{1}+2 \lambda_{2} \neq 0$ ), which is proportional to the identity matrix. This means symmetry breaking to $\mathrm{O}(5)$. Here the decay constant is defined as 
$f_{S}^{2} \equiv M^{2} /\left(10 \lambda_{1}+2 \lambda_{2}\right)$. For the vacuum expectation value

$$
\langle S\rangle=f_{S}\left(\begin{array}{lll} 
& & \mathbf{1}_{2} \\
& 1 & \\
\mathbf{1}_{2} & &
\end{array}\right)
$$

the global $\mathrm{SU}(5) \times \mathrm{U}(1)$ is broken to $\mathrm{SO}(5)$. Fluctuations around the vacuum expectation value (5) are parameterized as $S=\langle S\rangle+\bar{S}$ with

$$
\bar{S}=i N+R, \quad N=\left(\begin{array}{ccc}
\phi & h & \chi \\
h^{T} & K_{i} & h^{\dagger} \\
\chi^{T} & h^{*} & \phi^{\dagger}
\end{array}\right), \quad R=\left(\begin{array}{ccc}
\Phi & H & X \\
H^{T} & K_{r} & H^{\dagger} \\
X^{T} & H^{*} & \Phi^{\dagger}
\end{array}\right) .
$$

which are composed of the two $2 \times 2$ complex symmetric tensors $\phi$ and $\Phi$, the two complex doublet fields $h$ and $H$, the two $2 \times 2$ Hermite tensors $\chi$ and $X$ and the two real fields $K_{i}$ and $K_{r}$. The fields in the matrix $R$ have large masses $\sim M$.

After integrating out heavy fields, we obtain the Lagrangian term of effective vertex of gauge-scalar couplings $V_{\text {eff }}$ as [10]

$$
\begin{aligned}
-V_{\text {eff }}= & \left(\frac{1}{4} g^{2} W_{1}^{a} W_{2}^{a}+\frac{1}{8} g^{\prime 2} B_{1} B_{2}\right) h^{\dagger} h+\frac{1}{4} g^{2} W_{1}^{a} W_{2}^{a}\left(\operatorname{Tr}\left[\phi \phi^{\dagger}\right]+\operatorname{Tr}\left[\chi^{2}\right]\right) \\
& +\frac{1}{400} g^{\prime 2}\left(B_{1}^{2}+B_{2}^{2}+49 B_{1} B_{2}\right) \operatorname{Tr}\left[\phi \phi^{\dagger}\right]-\frac{3}{50} g^{\prime 2}\left(B_{1}-B_{2}\right)^{2} \operatorname{Tr}\left(\chi^{2}\right) .
\end{aligned}
$$

The Higgs field $h$ has gauge couplings of the form given in Eq. (2). Thus for the mass of the Higgs field, there is no quadratic divergence from gauge boson loop. On the other hand, $\operatorname{Tr}\left(\phi \phi^{\dagger}\right), \operatorname{Tr}\left(\chi^{2}\right)$ have interacting terms with $B_{1}^{2}$ and $B_{2}^{2}$. Due to these $\mathrm{U}(1)$ factors, the masses of $\phi$ and $\chi$ receive quadratic divergence.

As another aspect, we study the case in which the two breaking sectors in higher energy scales are spatially separated. At $y=0, \mathrm{SU}(5)$ is broken to $[\mathrm{SU}(2) \times \mathrm{U}(1)]^{2}$ and at $y=L, \mathrm{SU}(5)$ is broken to $\mathrm{SO}(5)$. Here $y$ is the coordinate of the fifth dimension and the fundamental region is $0 \leq y \leq L$. We assume that the five-dimensional spacetime is flat. It has been shown that at a single boundary $\mathrm{SU}(5)$ cannot be broken to $[\mathrm{SU}(2) \times \mathrm{U}(1)]$ by boundary conditions unless additional scalar fields are introduced [11]. It is needed to examine the consistency of possible boundary conditions to yield gauge symmetry breaking $\mathrm{SU}(5) \rightarrow[\mathrm{SU}(2) \times \mathrm{U}(1)]^{2}$ at $y=0$ and $\mathrm{SU}(5) \rightarrow \mathrm{SO}(5)$ at $y=L$. In order to realize the symmetry breaking above, we assign Neumann condition for the gauge bosons of the generators $T_{1}, T_{2}, T_{3}, T_{8}, T_{15}, T_{22}, T_{23}, T_{24}$ at $y=0$ and $T_{\overline{1}}, \cdots, T_{\overline{10}}$ given by $T_{\overline{1}}=\frac{1}{\sqrt{2}}\left(T_{1}-T_{22}\right), T_{\overline{2}}=\frac{1}{\sqrt{2}}\left(T_{2}+T_{23}\right), T_{\overline{3}}=\frac{1}{\sqrt{2}}\left(T_{4}-T_{13}\right), T_{\overline{4}}=\frac{1}{\sqrt{2}}\left(T_{5}-T_{14}\right)$, $T_{\overline{5}}=\frac{1}{\sqrt{2}}\left(T_{6}-T_{20}\right), T_{\overline{6}}=\frac{1}{\sqrt{2}}\left(T_{7}-T_{21}\right), T_{\overline{7}}=\frac{1}{\sqrt{2}}\left(T_{11}-T_{16}\right), T_{\overline{8}}=\frac{1}{\sqrt{2}}\left(T_{12}-T_{17}\right), T_{\overline{9}}=$ $\frac{1}{\sqrt{2}}\left(T_{3}+\frac{\sqrt{6}}{4} T_{15}-\frac{\sqrt{10}}{4} T_{24}\right), T_{10}=\frac{1}{\sqrt{2}}\left(\frac{\sqrt{3}}{3} T_{8}+\frac{5 \sqrt{6}}{12} T_{15}+\frac{\sqrt{10}}{4} T_{24}\right)$, at $y=L$ and Dirichlet condition for the other gauge bosons. Only the fields with Neumann condition at both boundaries have zero modes. The generators of zero modes are $T_{\overline{1}}, T_{\overline{2}}, T_{\overline{9}}, T_{\overline{10}}$. These generators form $\mathrm{SU}(2) \times \mathrm{U}(1)$ algebra. The gauge transformation laws are written 
as $\delta A_{M}^{a}=\partial_{M} \varepsilon^{a}+g f^{a b c} A_{M}^{b} \varepsilon^{c}+g f^{a \hat{b} \hat{c}} A_{M}^{\hat{b}} \varepsilon^{\hat{c}}, \delta A_{M}^{\hat{a}}=\partial_{M} \varepsilon^{\hat{a}}+g f^{\hat{a} b \hat{c}} A_{M}^{b} \varepsilon^{\hat{c}}+g f^{\hat{a} b \hat{c}} A_{M}^{b} \varepsilon^{\hat{c}}$, where $a$ and $\hat{a}$ indicate the generators of the subgroup and the coset, respectively. At $y=0, a$ and $\hat{a}$ represent the indices for $[\mathrm{SU}(2) \times \mathrm{U}(1)]^{2}$ and $\mathrm{SU}(5) /[\mathrm{SU}(2) \times \mathrm{U}(1)]^{2}$, respectively. At $y=L, a$ and $\hat{a}$ represent the indices for $\mathrm{SO}(5)$ and $\mathrm{SU}(5) / \mathrm{SO}(5)$, respectively. The boundary conditions at $y=0$ and $y=L$ are collectively written with the $a$ and $\hat{a}$. The left- and right-hand sides in the transformation laws have the boundary conditions shown in Table 1. From Table 1, it is found that all the boundary conditions are

TABLE 1. The boundary conditions for $\mathrm{SU}(5) \rightarrow \mathrm{SU}(2) \times \mathrm{U}(1)$.

\begin{tabular}{|c|c|c|c|c|c|}
\hline & LHS & RHS & & LHS & RHS \\
\hline$A_{\mu}^{a}$ & $\mathrm{~N}$ & $\mathrm{~N}+\mathrm{NN}+\mathrm{DD}$ & $A_{\mu}^{\hat{a}}$ & $\mathrm{D}$ & $\mathrm{D}+\mathrm{DN}+\mathrm{ND}$ \\
\hline$A_{y}^{a}$ & D & $\mathrm{D}+\mathrm{DN}+\mathrm{ND}$ & $A_{y}^{\hat{a}}$ & $\mathrm{~N}$ & $\mathrm{~N}+\mathrm{NN}+\mathrm{DD}$ \\
\hline
\end{tabular}

consistent with the gauge transformations. The result of the consistency in this case is also seen from the fact that Neumann and Dirichlet conditions imposed at each boundary can be assigned by the automorphism of orbifolds.

We have derived all the couplings of scalar-gauge interactions relevant to scalar mass corrections via gauge boson loop in high energy models. We have found that while the Higgs fields are protected with the coupling of the form (1) from having quadratic divergence, the other fields receive quadratic divergence through loop of the $\mathrm{U}(1)$ gauge boson. We have also considered a possibility of gauge symmetry breaking by boundary conditions. In our assignment for boundary conditions, the same gauge symmetry breaking as in the vacuum expectation value is produced in a consistent way with local gauge transformations.

\section{ACKNOWLEDGMENTS}

This work is supported by Scientific Grants from the Ministry of Education and Science, Grant No. 20244028.

\section{REFERENCES}

1. N. Arkani-Hamed, A. G. Cohen and H. Georgi, Phys. Lett. B 513, 232 (2001) [arXiv:hep-ph/0105239].

2. N. Arkani-Hamed, A. G. Cohen, E. Katz and A. E. Nelson, JHEP 0207, 034 (2002) [arXiv:hep$\mathrm{ph} / 0206021]$.

3. D. E. Kaplan and M. Schmaltz, JHEP 0310, 039 (2003) [arXiv:hep-ph/0302049].

4. H. C. Cheng and I. Low, JHEP 0309, 051 (2003) [arXiv:hep-ph/0308199].

5. I. Low, JHEP 0410, 067 (2004) [arXiv:hep-ph/0409025].

6. For a review see M. Schmaltz and D. Tucker-Smith, Ann. Rev. Nucl. Part. Sci. 55, 229 (2005) [arXiv:hep-ph/0502182].

7. C. Csaki, J. Heinonen, M. Perelstein and C. Spethmann, arXiv:0804.0622 [hep-ph].

8. S. R. Coleman, J. Wess and B. Zumino, Phys. Rev. 177, 2239 (1969).

9. C. G. . Callan, S. R. Coleman, J. Wess and B. Zumino, Phys. Rev. 177, 2247 (1969).

10. N. Uekusa, Phys. Rev. D 79, 085010 (2009) [arXiv:0810.5024 [hep-ph]].

11. N. Sakai and N. Uekusa, Prog. Theor. Phys. 118, 315 (2007) [arXiv:hep-th/0604121]. 